\title{
Ciclo anual del nitrógeno y el fósforo en el embalse Paso Bonito, Cienfuegos, Cuba
}

\author{
Carmen Betancourt ${ }^{1, *}$, Roberto Suárez ${ }^{2}$ y Liliana Toledo ${ }^{1}$ \\ ${ }^{1}$ Centro de Estudios Ambientales de Cienfuegos. Calle 17, esq. Ave 46 s/n, Reparto Reina, Cienfuegos 55100, \\ Cuba. \\ ${ }^{2}$ Universidad de Cienfuegos. Carretera a Rodas km 3. C.P. 55100 Cienfuegos, Cuba. \\ * Autor responsable de la correspondencia: carmen@gestion.ceac.cu
}

Recibido: 28/6/08 Aceptado: 12/11/08

\begin{abstract}
Nitrogen and phosphorus annual cycle in the Paso Bonito reservoir (Cienfuegos, Cuba)

The Paso Bonito reservoir is a source of supply for the city of Cienfuegos and part of the city of Santa Clara. Concentrations of nitrogen and phosphorous in the reservoir and their relationships with the watershed and reservoir management were analyzed on seven dates between September 2006 and November 2007. The reservoir's management was evaluated with respect to water influxes and discharges and the water residence time. In the water column near the reservoir discharge intake the ratio of dissolved inorganic nitrogen to phosphorus indicated that phytoplankton growth was most commonly co-limited by nitrogen and phosphorus or by phosphorus alone. Fifty-two percent of the phosphorous measurements classified the reservoir as eutrophic. Only $\mathrm{N}-\mathrm{NH}_{4}$ and $\mathrm{P}_{T}$ concentrations changed significantly with depth. Eighty seven percent of the $\mathrm{N}_{-} \mathrm{NO}_{3}$ values, as well as $\mathrm{N}_{-} \mathrm{NH}_{4}$ during the month of October, indicated water contamination according to the Cuban Regulation used. These results were expected due to watershed and reservoir management. Finally, some issues related to reservoir management are discussed.
\end{abstract}

Key words: Nitrogen, phosphourous, reservoir, watershed, Paso Bonito.

\section{RESUMEN}

\section{Ciclo anual del nitrógeno y el fósforo en el embalse Paso Bonito (Cienfuegos, Cuba)}

El embalse Paso Bonito, es fuente de abastecimiento de la ciudad de Cienfuegos y parte de la ciudad de Santa Clara. Las concentraciones de nitrógeno y fósforo en el embalse y sus relaciones con la cuenca vertiente y el tipo de gestión fueron evaluadas en una campaña de siete muestreos efectuados entre Septiembre de 2006 y Noviembre de 2007. En la columna de agua del punto de toma del embalse la relación entre el nitrógeno inorgánico disuelto y el fósforo total indican que el crecimiento del fitoplancton fue comúnmente limitado por nitrógeno y fósforo o por fósforo únicamente. El $52 \%$ de las mediciones de fósforo clasifican al embalse como eutrófico. Tan sólo las concentraciones de $\mathrm{N}-\mathrm{NH}_{4}$ y $\mathrm{P}_{\mathrm{T}}$ cambian significativamente con la profundidad. El $87 \%$ de los valores de $\mathrm{N}-\mathrm{NO}_{3}$ y del $\mathrm{N}-\mathrm{NH}_{4}$ del mes de Octubre indica contaminación del agua de acuerdo con la Regulación Cubana que se utiliza. Estos resultados eran los esperados debido a la gestión de la cuenca y del embalse. Finalmente, se dicuten algunos aspectos de la gestión del embalse.

Palabras clave: Nitrógeno, fósforo, embalse, cuenca, Paso Bonito. 


\section{INTRODUCCIÓN}

La eutrofización de los embalses constituye un serio problema ambiental teniendo en cuenta los múltiples usos de sus aguas. Puede producir alteración de las propiedades organolépticas del agua, corrosión de equipos hidroeléctricos, trastornos en los procesos de tratamiento por disminución del contenido de oxígeno, acumulación del amoníaco en la columna de agua y resuspensión de ciertos metales (Fe, Mn), además de afectar la dinámica ecológica de los cuerpos de agua (CEPIS, 2001).

Los embalses son altamente dependientes de los procesos que ocurren en su entorno. Su estado, así como las comunidades que lo habitan, son en su gran mayoría, una consecuencia de las características de la cuenca de drenaje y de las actividades que en ella se realizan (Seo \& Canale, 1999; Wetzel, 2001). Su estado trófico depende fundamentalmente de la carga de nutrientes que recibe, de su morfometría y del tiempo de residencia del agua en el mismo (Vollenweider, 1969, Dillon \& Rigler, 1975, Salmaso et al., 2003).

Los procesos de estratificación térmica y mezcla están vinculados con la respuesta del embalse. La forma de la cubeta y punto de desagüe son fundamentales, porque determinan el tipo de mezcla en la masa de agua.

El embalse Paso Bonito es utilizado como fuente de abastecimiento de la ciudad de Cienfuegos al centro Sur de Cuba y una parte de la ciudad de Santa Clara. Por su importancia tanto social como económica es objeto de investigación. Durante los años 2006 y 2007, se estudiaron los patrones de la temperatura, oxígeno disuelto, potencial redox y $\mathrm{pH}$ (Betancourt et al., 2009).

En este estudio el embalse presentó un patrón polimíctico durante el año 2007 provocado por lluvias intensas, mientras que durante el 2006 (año caracterizado por escasas precipitaciones), se observó estratificación térmica durante todo el verano, a pesar del embalse recibir volúmenes de agua 2.6 veces superior a su capacidad de almacenamiento. El patrón de distribución de oxígeno disuelto fue de tipo clinógrado, con potenciales redox negativos y altos valores de hierro durante la estratificación térmica del embalse (Betancourt et al., 2009).

El presente trabajo tiene como objetivo determinar las concentraciones de fósforo y nitrógeno en la superficie, medio y fondo de la columna de agua del punto de toma y vincular el comportamiento de estos elementos con aspectos referidos al manejo de la cuenca y del embalse.
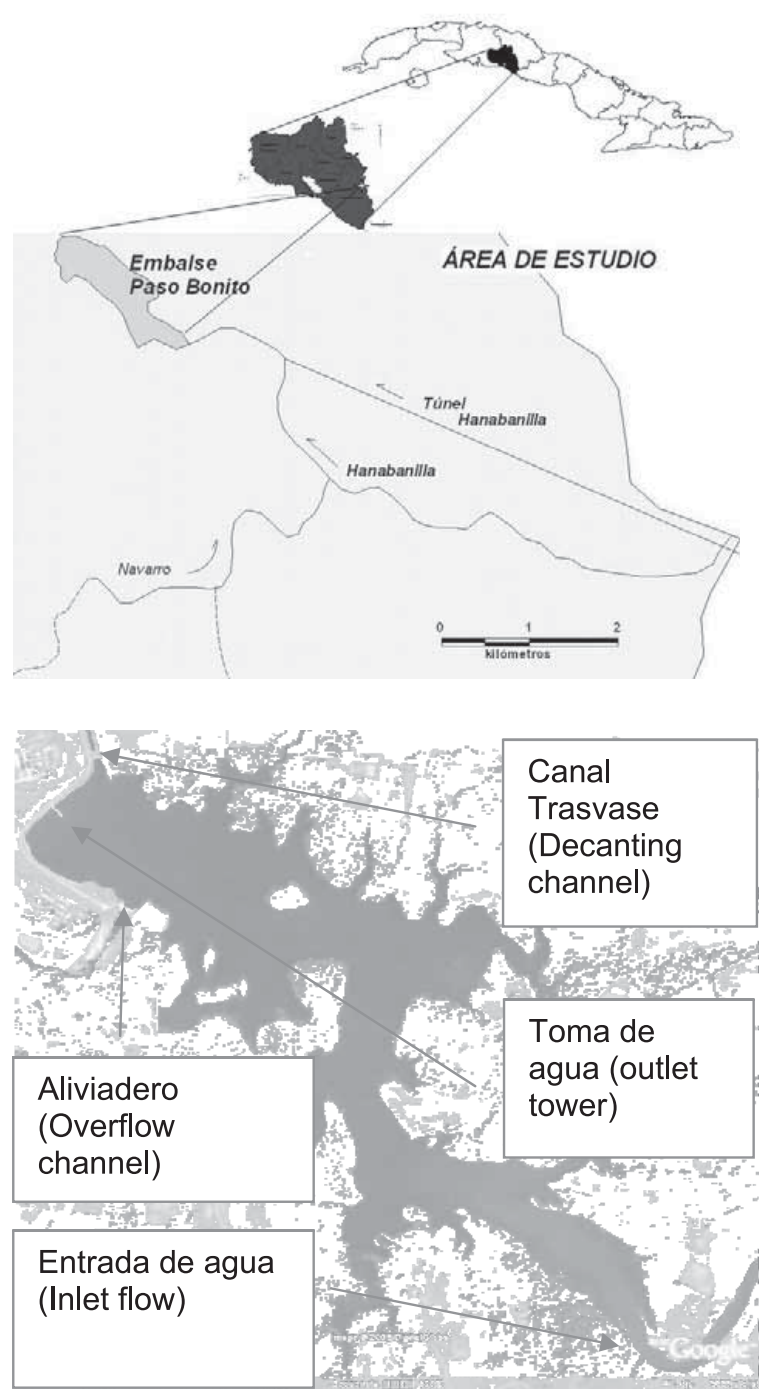

Figura 1. Localización del área de estudio. Location of the study area. 


\section{MATERIALES Y MÉTODOS}

\section{Área de estudio}

El Embalse Paso Bonito se ubica al Sureste de la provincia de Cienfuegos, región Centro-Sur de Cuba (Fig. 1) y fue construido en 1975. En este embalse se acumulan las aguas procedentes del río Hanabanilla, al cual se le incorpora el arroyo Navarro con abundantes sedimentos, y las aguas procedentes de una hidroeléctrica que se alimenta del embalse Hanabanilla a través de un túnel.

Su cuenca está ubicada en una zona de montaña y premontaña, lo cual facilita la erosión y el transporte de contaminantes. En la Tabla 1 se muestran algunas características morfométricas de la cuenca y del embalse.

\section{Muestreo y ensayos}

Se realizaron siete muestreos a partir de Septiembre de 2006 hasta Noviembre de 2007, durante los meses de principio y final de los períodos de mezcla (Noviembre, Diciembre, Febrero, Mayo) y estratificación térmica (Septiembre, Octubre, Julio). Las muestras se tomaron en los niveles superficie $(0 \mathrm{~m})$, medio $(5 \mathrm{~m})$ y fondo $(10 \mathrm{~m})$ en la columna de agua del punto de toma del embalse, con una botella Niskin de 5 litros de capacidad.
Las concentraciones de nitrógeno amoniacal (N$\mathrm{NH}_{4}$ ) se determinaron por formación de indofenol azul, el nitrógeno de nitrito $\left(\mathrm{N}-\mathrm{NO}_{2}\right)$ por diazotación con sulfanilamida, el nitrógeno de nitrato $\left(\mathrm{N}-\mathrm{NO}_{3}\right)$ por reducción con hidracina, el fósforo de ortofosfato $\left(\mathrm{P}-\mathrm{PO}_{4}\right)$ se cuantificó por formación de un complejo azul con molibdato de amonio y el fósforo total $\left(\mathrm{P}_{T}\right)$ por reducción con ácido ascórbico. Todos los ensayos se realizaron según las especificaciones de APHA (1998).

Los ensayos se realizaron sin filtrar las muestras por lo que incluyen los nutrientes suspendidos y disueltos. Los límites de cuantificación (LC) expresados en $\mathrm{mg} / \mathrm{l}$, fueron $\mathrm{N}-\mathrm{NH}_{4} ; 0.045$, $\mathrm{N}-\mathrm{NO}_{2} ; 0.001, \mathrm{~N}-\mathrm{NO}_{3} ; 0.006, \mathrm{P}-\mathrm{PO}_{4} ; 0.005$, $\mathrm{P}_{T} ; 0.013$. Los valores menores que el límite de cuantificación se consideraron cero para la confección de los gráficos.

\section{Otros procedimientos}

El cálculo de la relación $\mathrm{Ni}_{T}: \mathrm{P}_{T}$ se realizó considerando sólamente el nitrógeno inorgánico total $\left(\mathrm{Ni}_{T}\right)$. Las especies $\mathrm{N}-\mathrm{NO}_{3}$ y $\mathrm{N}-\mathrm{NH}_{4}$ se consideran las formas de nitrógeno biológicamente asimilables.

La clasificación del estado trófico se realizó según los criterios de OECD (1982), citado por CEPIS (2001). Esta clasificación considera oligotrofia para concentraciones menores que

Tabla 1. Características morfométricas del embalse según datos tomados del archivo de la Delegación de Recursos Hidráulicos en Cienfuegos. Morphometric characteristics for the reservoir according to data taken from the Delegación de Recursos Hidráulicos en Cienfuegos.

$\begin{array}{lc}\text { Área de la cuenca }\left(\mathrm{km}^{2}\right) & 65.0 \\ \text { Altitud media de la cuenca }(\mathrm{m}) & 187.0 \\ \text { Altitud del embalse }(\mathrm{m}) & 86.3 \\ \text { Volumen }\left(\mathrm{hm}^{3}\right) & 8.0 \\ \text { Area }\left(\mathrm{km}^{2}\right) & 1.25 \\ \text { Cota del canal trasvase }(\mathrm{msnm}) & 79.75 \\ \text { Cota punto de toma (msnm) } & 76.5 \\ \text { Diferencia de altura entre el canal trasvase y el punto de toma (m) } & 3.25 \\ \text { Escorrentía media anual }\left(\mathrm{hm}^{3}\right) & 37.6 \\ \text { Longitud del río principal }(\mathrm{km}) & 16.5 \\ \text { Profundidad máxima }(\mathrm{m}) & 19.5 \\ \text { Profundidad media }(\mathrm{m}) & 6.5 \\ \text { Línea de costa }(\mathrm{km}) & 9.56\end{array}$


$10 \mu \mathrm{g} / \mathrm{l}$ de $\mathrm{P}_{T}$, mesotrofia para un intervalo de 10-30 $\mu \mathrm{g} / \mathrm{l}$, eutrofia entre 35-100 $\mu \mathrm{g} / \mathrm{l}$ e hipertrofia para valores superiores a $100 \mu \mathrm{g} / \mathrm{l}$. Los criterios incluyen además valores de clorofila $a$, transparencia y fósforo para la clasificación trófica de los lagos y embalses. En este caso particular sólo se consideró el criterio referido al fósforo.

Se siguió el criterio propuesto por Morris \& Lewis (1988) para evaluar la relación $\mathrm{Ni}_{T}: \mathrm{PT}$ (Nitrógeno inorgánico disuelto:Fósforo total), como indicador del nutriente que limita la productividad del sistema. Estos autores consideran limitaciones de nitrógeno cuando la relación es menor que 0.5, para el intervalo 0.5-4.0, ambos limitan y para valores superiores a 5 es el fósforo el nutriente limitante.

Para establecer la existencia de interacciones significativas de dos variables seleccionadas sobre una tercera variable de interés, se aplicó análisis de varianza factorial y unifactorial a todas las variables en estudio, verificando en cada uno el cumplimiento de los supuestos y aplicando en los casos posibles las alternativas no paramétricas; en los casos en los que no se cumplieron los requisitos de los análisis, se realizó el análisis descriptivo de los resultados, con el apoyo de los gráficos de perfiles.

La calidad del agua se evaluó mediante la Norma Cubana (NC), (1986) que regula los requisitos de las fuentes de abastecimiento para el consumo humano. Esta Norma considera que concentraciones de $\mathrm{N}-\mathrm{NH}_{4}$ y de $\mathrm{N}-\mathrm{NO}_{2}$ superiores a $0.388 \mathrm{mg} / \mathrm{l}$ y $0.003 \mathrm{mg} / \mathrm{l}$ respectivamente, indican contaminación del agua.

Para identificar los problemas en el manejo de la cuenca se realizó un diagnóstico que incluyó, fundamentalmente, recorridos y entrevistas a trabajadores y pobladores del lugar, así como recopilación de información de las diferentes instituciones ubicadas en la misma. La información relacionada con el manejo del embalse se obtuvo de la base de datos de la Dirección del Instituto de Recursos Hidráulicos. A partir del número de animales existentes en la cuenca se calculó la carga contaminante aportada según los criterios de Castagnino (1982) (Tabla 2).
Tabla 2. Cargas de $\mathrm{N}_{T}$ y $\mathrm{P}_{T}$ producidas por cada animal de los principales tipos de ganado (Datos según Castagnino (1982). Per capita $N_{\mathrm{T}}$ and $P_{\mathrm{T}}$ loads of common types of livestock (Data from Castagnino (1982).

\begin{tabular}{lcc}
\hline & $\mathrm{P}_{T} *$ & $\mathrm{~N}_{T}{ }^{*}$ \\
\hline Ganado vacuno (g/animal-año) & 7000 & 54.8 \\
Ganado porcino (g/animal-año) & 3000 & 14.6 \\
\hline
\end{tabular}

\section{RESULTADOS}

\section{Nitrógeno y fósforo en la columna de agua del embalse}

\section{Nitrógeno inorgánico}

El intervalo de $\mathrm{Ni}_{T}$ encontrado en el embalse osciló entre 0.007 y $0.406 \mathrm{mg} / \mathrm{l}$ (Fig. 2), el valor más alto se observó en el fondo en el mes de Octubre de 2006, al final del período de estratificación térmica y el más bajo, en la superficie en Octubre de 2007, después de intensas lluvias que provocaron la mezcla del embalse.

Las concentraciones de $\mathrm{N}-\mathrm{NO}_{3}$ estuvieron en el intervalo de 0.006-0.921 mg/l, los valores más

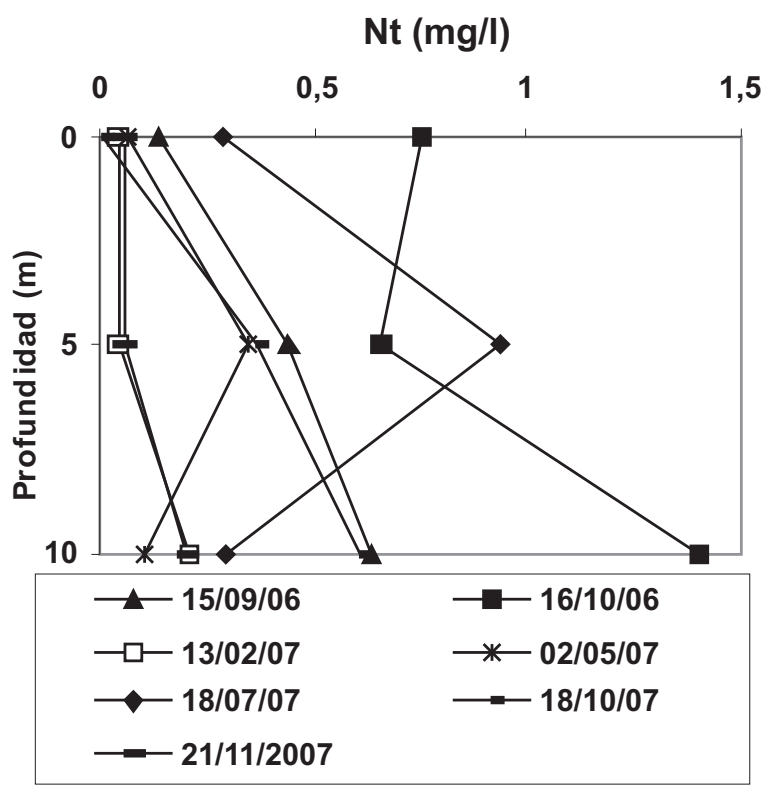

Figura 2. Perfiles de nitrógeno inorgánico total durante el periodo de estudio. Total inorganic nitrogen profiles during the study. 


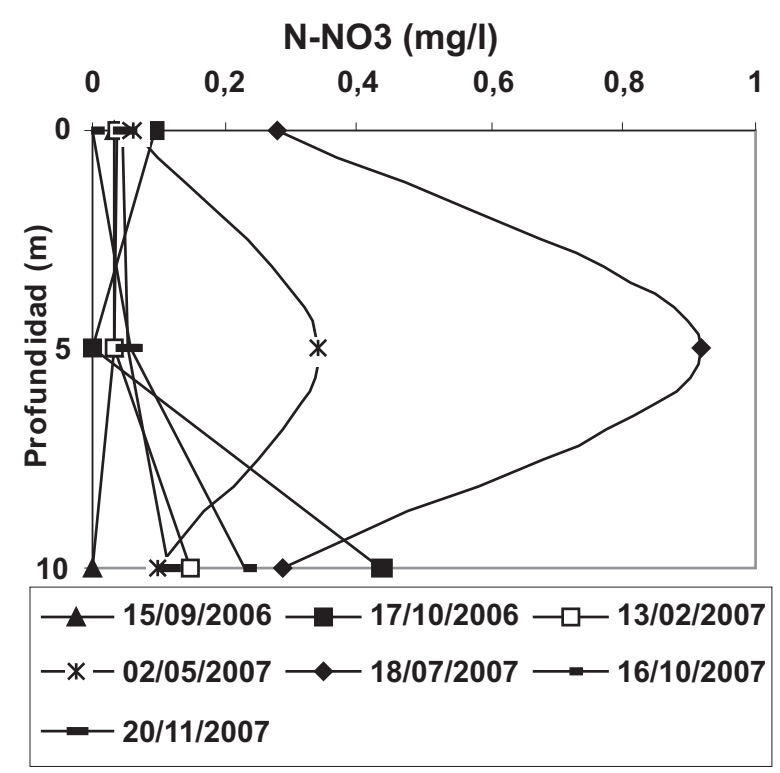

Figura 3. Evolución temporal de los perfiles de nitrato. Temporal changes in nitrate profiles.

elevados se registraron en el fondo, excepto en el mes de Mayo y Julio que se localizaron en la profundidad intermedia. Para el resto de los meses se observaron concentraciones similares entre la superficie y medio. (Fig. 3).

El N-NO ${ }_{2}$ siempre estuvo presente en el agua en el intervalo de 0.001-0.039 mg/l. En Octubre de 2007 se detectaron los valores más elevados de la etapa en estudio, incrementándose gradualmente hacia el fondo (Fig. 4).

El $87 \%$ de las mediciones realizadas son mayores que $0.003 \mathrm{mg} / \mathrm{l}$, valor que indica contaminación del agua según la Norma Cubana (NC) (1986). Los registros más bajos fueron en Febrero, mientras circulaba el embalse aunque no se observó una regularidad en su comportamiento.

Para el N- $\mathrm{NH}_{4}$ se detectaron valores en el intervalo de 0.045-0.961 mg/l, los valores más altos se encontraron en el fondo. Durante los meses de Septiembre de 2006 y Octubre de 2007 se registraron las mayores concentraciones (Fig. 5), que correspondieron con el período de una estratificación térmica marcada y estable, según Betancourt et al., (2009).

En el mes de Septiembre a partir de una profundidad de $5 \mathrm{~m}$, la concentración registrada indicó contaminación del agua según NC, (1986), fe-

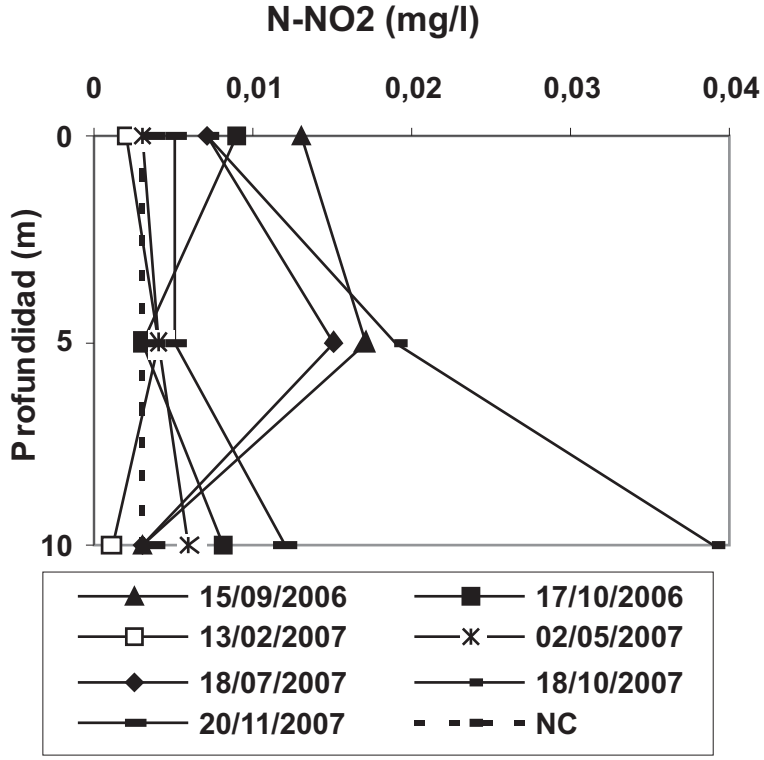

Figura 4. Evolución temporal de los perfiles de nitrito. Temporal changes in the nitrite profiles.

nómeno que se registra en toda la columna en el mes de Octubre de 2006; en estos meses el nitrógeno amoniacal aporta una mayor contribución al $\mathrm{Ni}_{T}$, alcanzando proporciones entre el $56 \%$ y el $99.55 \%$.

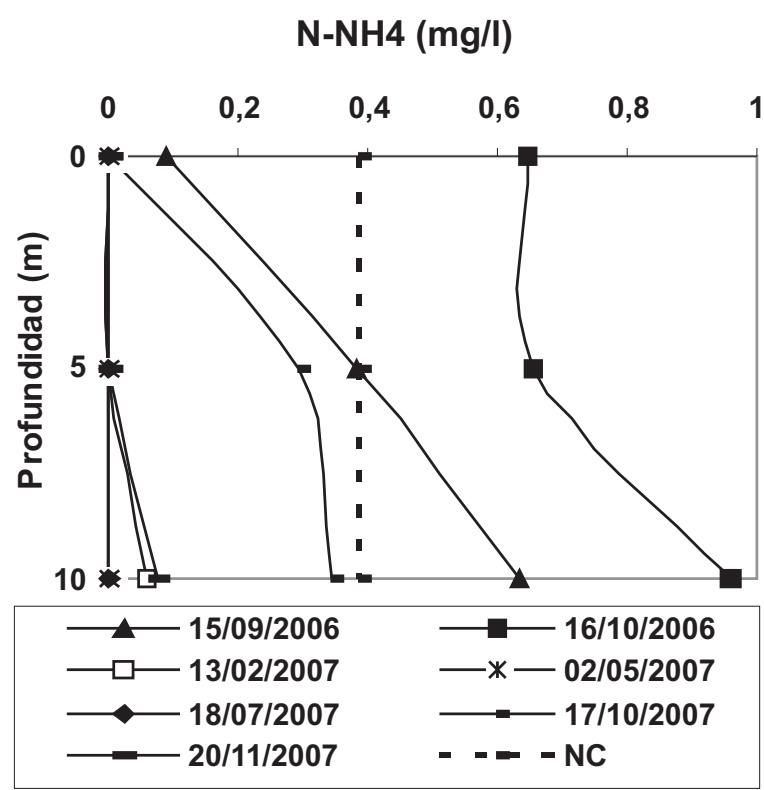

Figura 5. Evolución temporal de los perfiles de amonio. Temporal changes in the ammonia profiles. 


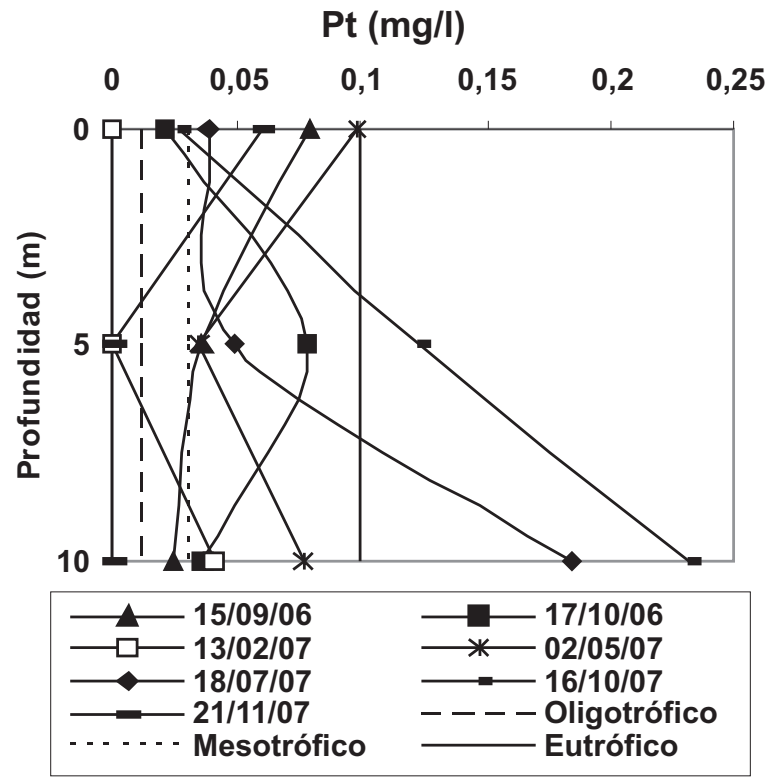

Figura 6. Evolución temporal de los perfiles de fósforo total. Temporal changes in the total phosphorus profiles.

\section{Fósforo}

El $19 \%$ de las mediciones de $\mathrm{P}_{T}$ clasificó al embalse como oligotrófico, el $19 \%$ mesotrófico, el $52 \%$ eutrófico y el $14 \%$ hipertrófico, según los criterios de OECD, (1982). Este indicador fue superior al $\mathrm{P}_{-} \mathrm{PO}_{4}$, lo cual implica la presencia de otras especies químicas de fósforo. El intervalo de valores registrados se ubicó entre 0.013-0.232 mg/l y las concentraciones más altas se registraron en el fondo del embalse. (Fig. 6).

\section{Interacción entre el fósforo total, nitrógeno amoniacal y profundidad}

En el estudio de las interacciones entre las variables evaluadas sólo resultó de interés la interacción del $\mathrm{N}-\mathrm{NH}_{4}$ a diferentes profundidades sobre el $\mathrm{P}_{T}$. En la figura 7 se muestra que los valores medios más altos de $\mathrm{P}_{T}$ corresponden al fondo del embalse, registrándose el valor más alto cuando las concentraciones de $\mathrm{N}-\mathrm{NH}_{4}$ estuvieron en el intervalo comprendido entre 0.001-0.445 mg/l. Para concentraciones de amonio superiores a $0.445 \mathrm{mg} / \mathrm{l}$, los valores medios de $\mathrm{P}_{T}$ en las profundidades disminuyeron hasta cifras similares a las registradas en la superficie. Estos valores de

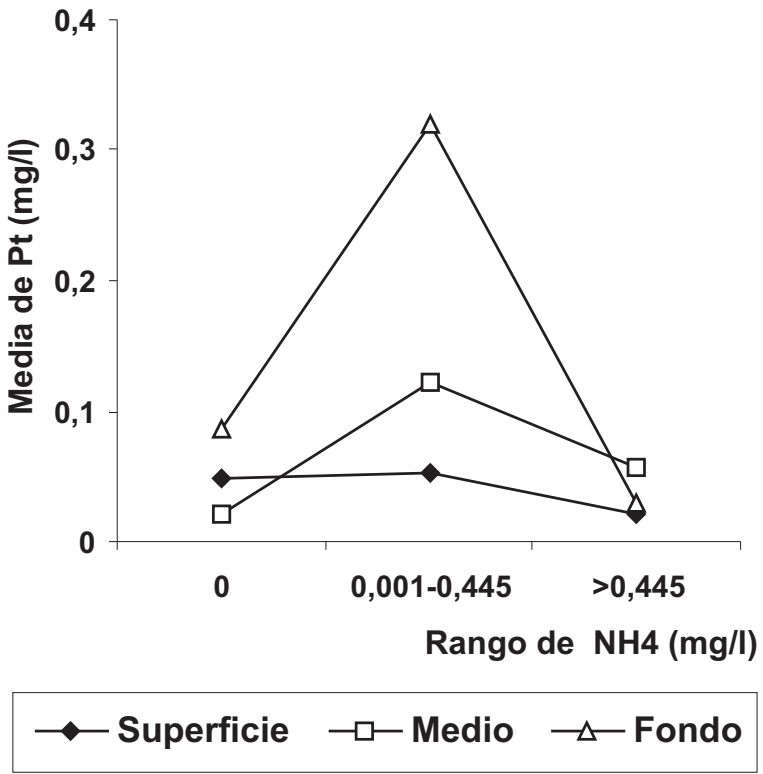

Figura 7. Relaciones por profundidades entre el amonio y el fósforo total. Relationships by depths between ammonia and total phosphorus.

amonio se alcanzaron durante el período en que el embalse permaneció estratificado térmicamente, cuando se liberaron altas concentraciones de hierro (Betancourt et al., 2009) que pudieron provocar la precipitación del fósforo.

El $\mathrm{P}_{-} \mathrm{PO}_{4}$ estuvo en el intervalo de 0.005$0.172 \mathrm{mg} / \mathrm{l}$. (Fig. 8). Sólo se detectó en la columna de agua en los meses de Octubre de 2007, Mayo, Septiembre y en la superficie, en el mes Julio. Sus bajos valores coincidieron con altas concentraciones de hierro.

\section{Relación $\mathrm{Ni}_{T}: \mathrm{P}_{T}$}

Considerando el criterio propuesto por Morris \& Lewis (1988), el $52.4 \%$ de las mediciones estuvo limitado por el fósforo, el $42.9 \%$ por ambos nutrientes y sólo una medición realizada en la superficie registró limitación por nitrógeno (Fig. 9). La limitación por fósforo predomina en el fondo e intermedio, y por nitrógeno, en la superficie.

En los meses de Febrero, Mayo, Noviembre y Octubre de 2007 fueron registrados procesos de mezcla en el embalse (Betancourt et al., 2008), observándose con mayor predominancia, la limitación por ambos nutrientes, mientras que en los 

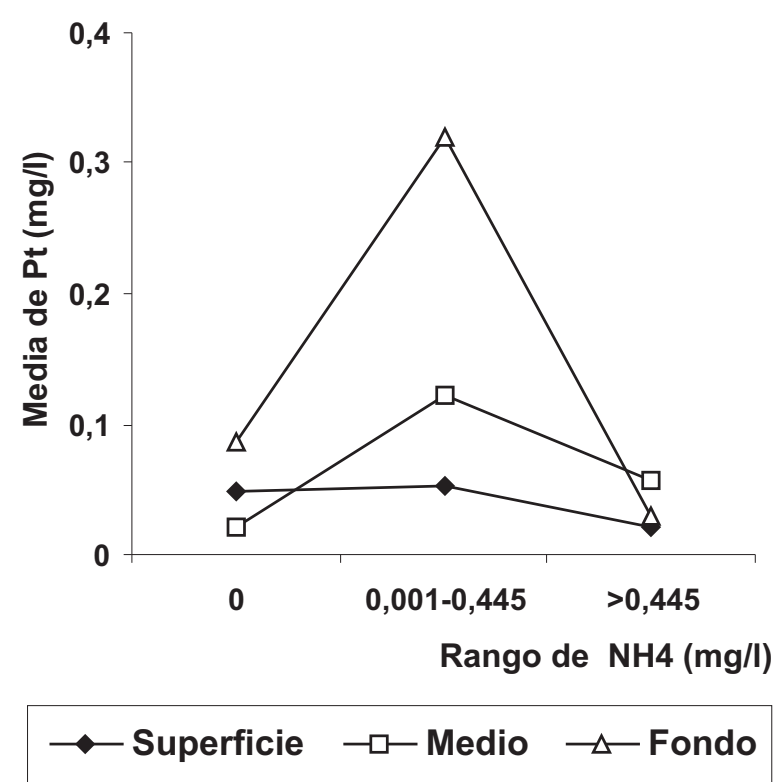

Figura 8. Evolución temporal de los perfiles de fosfato durante el periodo estudiado. Temporal changes in the phosphate profiles during the study.

meses que ocurrió estratificación térmica, predominó la limitación por fósforo.

\section{Entrada y salida de agua al embalse}

Las entradas y salidas de agua en el embalse se muestran en las figuras 10 y 11 respectivamente. El mayor aporte de agua procede de la generación de energía, (159.5 hm $\left.\mathrm{hm}^{3} / \mathrm{año}\right)$, mientras que la escorrentía $\left(35.5 \mathrm{hm}^{3} / \mathrm{año}\right)$ representa porcentajes bajos del total de la entrada (menores que el $40 \%$ ). Esta baja escorrentía es una de las consecuencias de la construcción y explotación del embalse Hanabanilla ubicado aguas arriba de Paso Bonito.

Los mayores volúmenes de salidas (Fig. 11) corresponden con el trasvase de agua hacia el embalse Avilés (124.8 $\left.\mathrm{hm}^{3} / \mathrm{año}\right)$ a través de un canal. Otras salidas fueron la extracción para el consumo humano, estimados en $67.4 \mathrm{hm}^{3} / \mathrm{año}$, y el control de avenidas con un vertido de $10.4 \mathrm{hm}^{3} /$ año, el punto de salida más superficial del embalse corresponde con la que se utiliza para el trasvase de agua y está situada a $3.25 \mathrm{~m}$ por encima del punto de toma (Tabla 1).

El agua que se incorpora por la generación de energía, procede del embalse Hanabanilla y antes

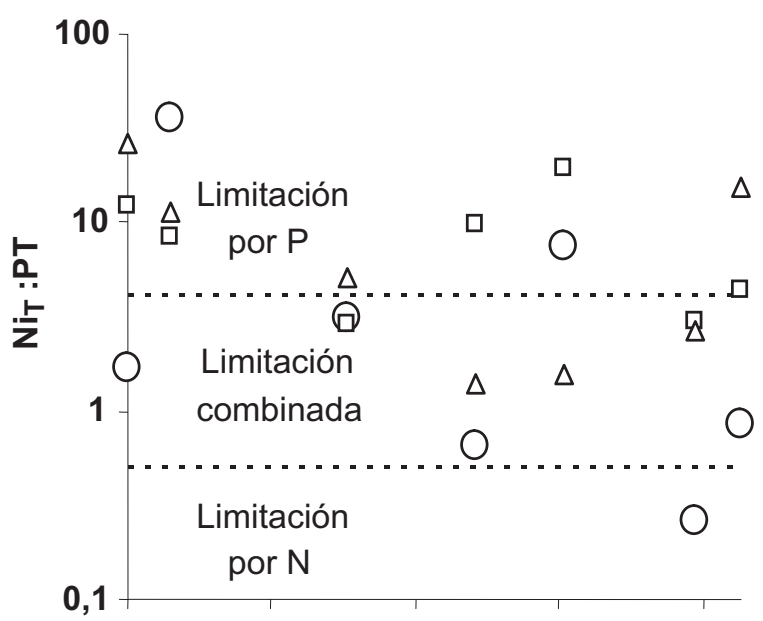

Sep-06 Dic-06 Mar-07 Jun-07 Oct-07

○ Superficie $\square$ Medio $\Delta$ Fondo

Figura 9. Relaciones entre el nitrógeno inorgánico y el fósforo total. Las líneas horizontales indican niveles de la razón de $\mathrm{Ni}_{T}$ y $\mathrm{P}_{T}$ sugeridos por Morris and Lewis (1988) para diferenciar entre limitación por nitrógeno o fósforo para el fitoplancton. Relationships between $N i_{\mathrm{T}}$ and $P_{\mathrm{T}}$. The horizontal lines indicate $D i_{\mathrm{T}}: T_{\mathrm{P}}$ ratios suggested by Morris and Lewis (1988) to differentiate between nitrogen and phosphorus limitation of phytoplankton.

de incorporarse al embalse Paso Bonito, es utilizada en una hidroeléctrica donde pudiera elevarse su temperatura. En su trayecto hacia el embalse se transporta por un túnel soterrado que podría facilitar la conservación del calor facilitando así su transporte superficial hacia el canal trasvase ubicado en un lateral del mismo (Fig. 1b)

La calidad de esta agua fue estudiada durante la década 1989-2000 por Sánchez (2000), determinándose valores de $\mathrm{Ni}_{T}$ en un intervalo de 0.280 $0.598 \mathrm{mg} / \mathrm{l}$ y de fósforo total $\left(\mathrm{P}_{T}\right)$ en el intervalo de 0.035-0.078 mg/l; lo cual indicó oligo-mesotrofia para el nitrógeno y eutrofia para el fósforo.

Para evaluar la influencia de la entrada de agua al embalse con la estabilidad del mismo, se compararon los volúmenes incorporados con el patrón de estratificación térmica (Betancourt et al., 2009), correspondiente a similar etapa de estudio, observándose que en Octubre de 2006 el embalse recibió $20.2 \mathrm{hm}^{3}$ del agua procedente de la generación, un valor 2.5 veces superior al volumen del embalse, mientras que la escorrentía fue baja $\left(0.135 \mathrm{hm}^{3}\right)$. Esta situación no produjo la ruptura de la termoclina. 

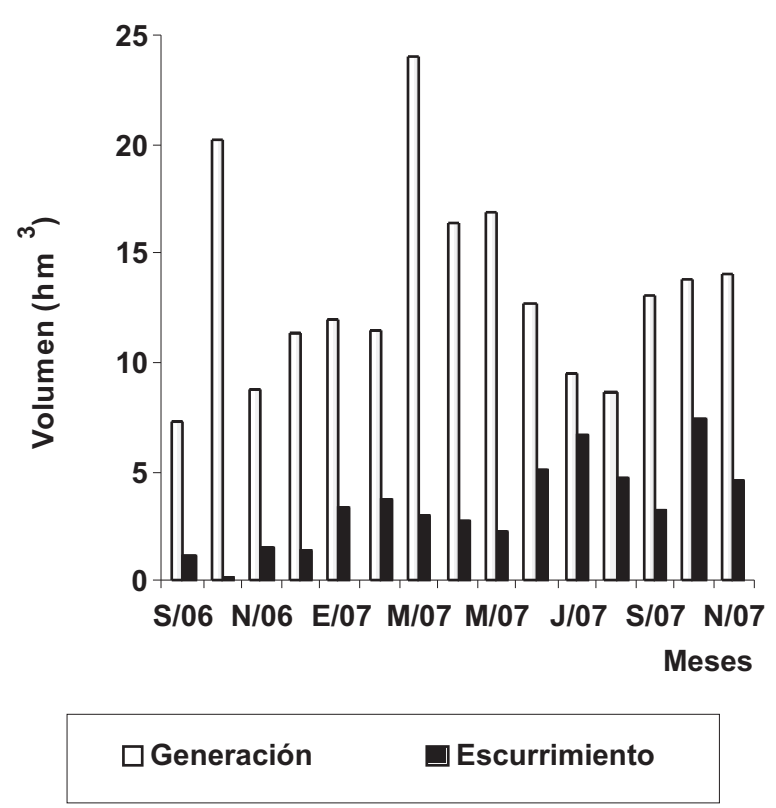

Figura 10. Volumen de agua entrado en el embalse según su origen. Water flow into the reservoir according to their source.

Sin embargo, en Octubre de 2007 la incorporación por generación fue de $13.8 \mathrm{hm}^{3}$ y la escorrentía ascendió a $7.44 \mathrm{hm}^{3}$, rompiéndose la termoclina y mezclándose el embalse. De lo anterior se puede deducir que, la estabilidad del embalse guarda una vinculación estrecha con su manejo y con la ocurrencia de precipitaciones abundantes. Cuando la escorrentía es pequeña y la entrada de agua por concepto de generación es grande, los altos volúmenes de salidas de agua (Fig. 11) que se producen por el canal trasvase ubicado en un lateral del embalse, pueden favorecer la circulación superficial y lateral, sin provocar la mezcla en la columna de agua del punto de toma que se localiza en el centro y cerca del muro de la presa.

El valor medio del tiempo de residencia del agua en el embalse (14.9 \pm 3.43 días) se obtuvo mediante el cociente del volumen de agua almacenada por el flujo de salida (Foy, 1992; Sivadier et al., 1994; Straskraba et al., 1995).

Según Wetzel (2001), tiempos de residencia similares se pueden clasificar como cortos favoreciendo los procesos de mezcla. Otros autores han encontrado estratificación térmica débil para tiempos de residencia de igual orden (Nadim et al., 2007). Sin embargo, a pesar del tiempo de

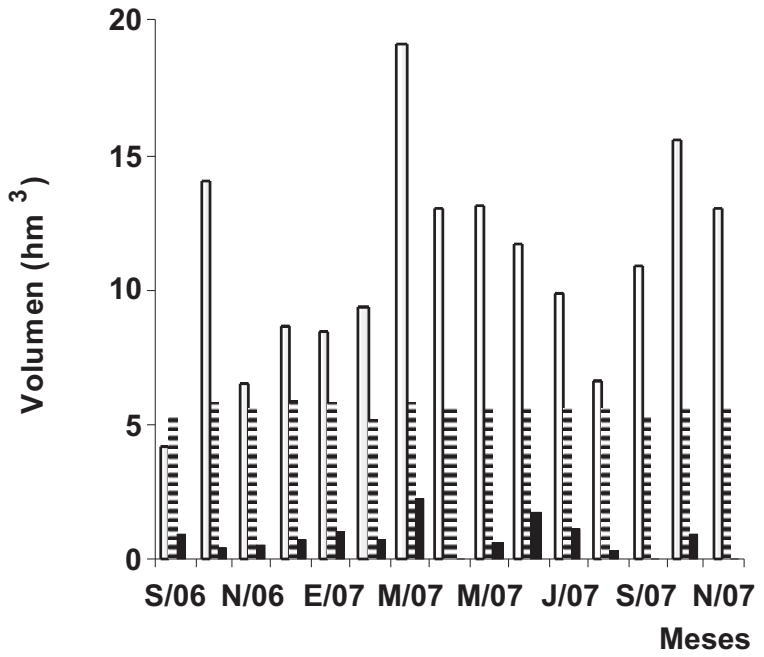

$\square$ Trasvase = Entrega $\square$ Vertimiento

Figura 11. Volumen de agua salido del embalse. Water discharges from the reservoir

residencia observado en este embalse, se pone de manifiesto una estratificación térmica estable en el punto de toma (Betancourt et al., 2009), que es interrumpida en el período estudiado, sólo cuando ocurren lluvias intensas.

\section{Procesos significativos identificados en el manejo de la cuenca}

Fuentes contaminantes en la cuenca Paso Bonito

1. Pequeñas poblaciones ( 608 hab. en total) que tratan sus residuales mediante fosa séptica y algunas viviendas que vierten directamente a corrientes superficiales, constituyen aporte de nitrógeno y fósforo.

Tabla 3. Estimación de la carga de nutrientes al embalse según el tipo de ganado. Estimation of the nutrient loads to the reservoir from each type of livestock.

\begin{tabular}{lcr}
\hline & N aportado kg/día & P aportado kg/día \\
\hline Porcino & $24 \mathrm{~kg} /$ día & $4.9 \mathrm{~kg} /$ día \\
Vacuno & $900 \mathrm{~kg} /$ día & $115.6 \mathrm{~kg} /$ día \\
Total & $924 \mathrm{~kg} /$ día & $120.5 \mathrm{~kg} /$ día \\
\hline
\end{tabular}




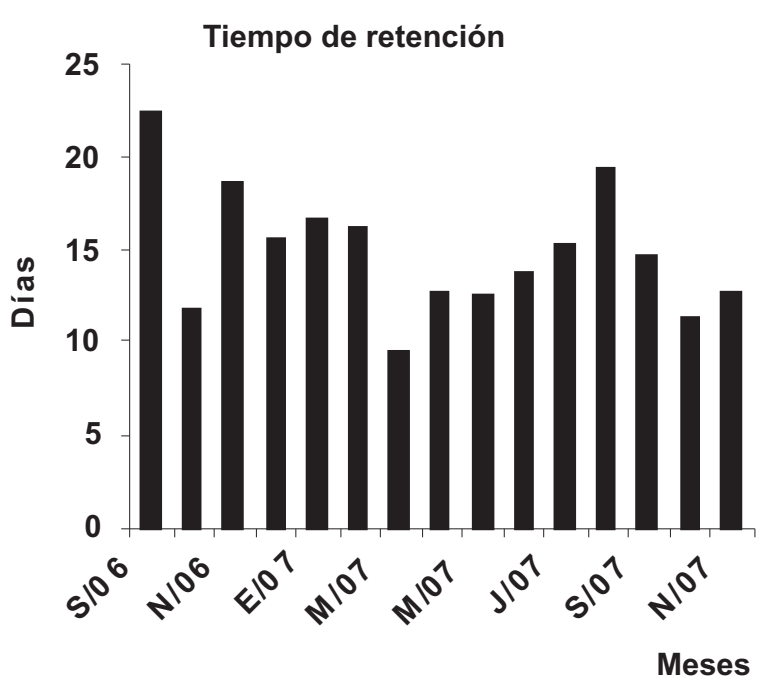

Figura 12. Tiempo de residencia del agua en el embalse. Water residence times in the reservoir.

2. Ganado vacuno y porcino cuyos residuales son parcialmente tratados. El ganado vacuno se cría de forma extensiva y constituye un aporte difuso. En la Tabla 3 se presenta la carga de fósforo y nitrógeno estimada.

3. Cultivo de tabaco en ambas márgenes del río Hanabanilla, con uso de fertilizantes y pesticidas. Presencia de cultivos temporales como el arroz, y otros permanentes (mango y naranja).

4. Predominio de cultivos a favor de la pendiente, lo que facilita la erosión y el arrastre de componente del suelo hacia el embalse.

5. Reparaciones, de caminos y carreteras con incumplimiento de las normas establecidas para el movimiento de tierra, que favorece la incorporación de sedimentos al embalse.

\section{DISCUSIÓN}

Los valores elevados de $\mathrm{N}-\mathrm{NH}_{4}$ y su mayor proporción respecto al $\mathrm{Ni}_{T}$, durante la estratificación térmica (Octubre 2006), pueden ser causados por su liberación desde los sedimentos, considerando que la escorrentía ese mes estuvo bastante deprimida (Fig. 10). Según Knox et al., (1981), la ma- yor proporción es considerada como un indicativo de nitrógeno reciclado procedente de procesos de mineralización desde los sedimentos; también se ha correlacionado con el estado trófico de los embalses (Beutel, 2006). Otros investigadores han planteado que en ausencia de oxígeno disuelto, como sucede en el fondo de este embalse (Betancourt, et al., 2009), las concentraciones liberadas desde el fondo aumentan (Al Bakrid \& Chowdhury, 2006, Beutel et al., 2008).

El fósforo, al igual que el amonio, puede ser liberado desde los sedimentos. Autores como Chowdhury \& Al bakri (2006), encontraron que la liberación de fósforo al agua puede representar hasta el $93 \%$ del total de fósforo incorporado a un embalse. En este trabajo se encontraron las concentraciones más altas de fósforo en el fondo, que también pudieran estar relacionadas con su liberación desde los sedimentos.

Las concentraciones de nitrógeno encontradas en la columna de agua durante la estratificación térmica, en el mes de Octubre de 2006, la clasificación trófica del $66 \%$ de las mediciones de $\mathrm{P}_{T}$ así como el déficit de oxígeno en la zona hipolimnética (Betancourt et al., 2009) y la aparición de especies clorofíceas, especialmente las que pertenecen al orden Chlorococcales, durante el año 2006, (Comas et al., 2007), apuntan a una clasificación eutrófica de las aguas del embalse Paso Bonito.

Considerando lo antes expuesto, así como la carga de nitrógeno y fósforo aportada por la cuenca (Tabla 3), y la calidad del agua que recibe procedente de la generación de energía, se hace necesario pensar en una correcta gestión, tanto en la cuenca de alimentación como en el embalse.

Recientemente se ha valorado por parte de la Gerencia del Agua en la provincia de Cienfuegos la ubicación de una toma de agua móvil para facilitar la succión del agua en las capas más superficiales durante períodos de afectaciones de la calidad de la misma. De esta forma disminuirían las dificultades con su tratamiento, y se garantizaría la entrega de un recurso de mayor calidad. Sin embargo, puede potenciar los efectos de eutrofización y comprometer aún más la calidad del agua para consumo humano a largo plazo. Se estima que en el mes de Octubre de 2007 se extra- 
jeron por el punto de toma ubicado a $7.73 \mathrm{~m}$ de profundidad, $0.605 \mathrm{t}$ de $\mathrm{Ni}_{T}$ y $1.800 \mathrm{t}$ de $\mathrm{P}_{T}$, carga de nutrientes extraídos que disminuiría considerablemente en caso que el punto de toma se trasladara para zonas más superficiales.

James et al. (2003), encontraron una relación inversa entre el tiempo de residencia y la concentración de $\mathrm{Ni}_{T}$, mientras que un trabajo de Maberly et al. (2003) revelaba que solamente el $20 \%$ de un grupo de lagos estudiados con tiempo de residencia corto tenían el fósforo limitante. Palau (2003) recomienda controlar los tiempos de residencia para el control de la eutrofización. En el caso del embalse estudiado, el tiempo de residencia corto (Fig. 12) no guarda relación con los procesos de mezcla y estratificación como se planteó en los resultados de este trabajo. Al parecer, el canal de trasvase ubicado en un lateral del embalse (Fig. 1b), en una posición más superficial que el punto de toma, con mayores volúmenes de salida, así como la entrada de porcentajes elevados de agua procedente de la generación de energía, con una temperatura que pudiera ser superior a la del embalse, parecen ser aspectos a tener en cuenta en su manejo si se desea que los procesos de mezcla se verifiquen con mayor frecuencia en la columna de agua de la obra de toma.

La extracción de agua procedente de capas superficiales con la toma móvil puede aumentar las concentraciones de $\mathrm{Ni}_{T}$ y $\mathrm{P}_{T}$ en el hipolimnion durante la estratificación térmica. Como consecuencia se puede producir un aumento de la concentración de estos nutrientes en la columna de agua durante los procesos de mezcla del embalse, que a su vez, estimularía el crecimiento del fitoplancton, aumentando la anoxia hipolimnética y con ello la liberación de nutrientes, materia orgánica y metales desde los sedimentos.

Recientemente se han detectado procesos que dificultan el tratamiento del agua para el consumo humano, por la resuspensión de materia orgánica, presencia de metales como el hierro y manganeso y también por la aparición de especies fitoplanctónicas como la diatomea Aulacoseira granulata que provocó obstrucciones en los filtros al final del verano de 2006 (Comas et al., 2007). Es evidente la necesidad de implementar medidas correctoras para que la eutrofi- zación se mantenga dentro de los límites permisibles. Para lograrlo es fundamental la reducción en el origen de los nutrientes.

Después de una disminución potencial de la carga externa, es necesario mitigar las concentraciones de nutrientes liberados desde los sedimentos. La instalación de una toma móvil es una buena práctica, pero debe estar combinada con extracciones de agua hipolimnética. Otra variante, aunque es costosa y puede provocar la resuspensión de sedimentos, es la oxigenación del agua hipolimnética.

El estudio adicional de la hidrodinámica del embalse, permitiría un conocimiento más integrado para su manejo y contribuiría a una mejor gestión del recurso. Además se hace necesario extender la implementación de medidas correctoras en la cuenca del embalse Hanabanilla, considerando que es la principal fuente de alimentación del embalse Paso Bonito.

\section{CONCLUSIONES}

Según las concentraciones de nitrógeno total el embalse se clasifica como mesotrófico con tendencia a la eutrofia cuando ocurre la estratificación térmica, mientras que el $52 \%$ de las mediciones de $\mathrm{P}_{T}$ clasifican al embalse como eutrófico.

El $52 \%$ de las mediciones estuvo limitado por el fósforo, el $43 \%$ por ambos nutrientes y sólo una medición realizada en la superficie registró limitación por nitrógeno, predominando la limitación por ambos nutrientes durante la verificación de procesos de mezcla en el embalse.

Se encontró interacción entre el nitrógeno amoniacal y el $\mathrm{P}_{T}$ a diferentes profundidades.

La aplicación de prácticas inapropiadas en la cuenca, provoca incorporación de nutrientes al embalse con afectaciones en la calidad del agua.

El tiempo de residencia del agua en el embalse es corto; sin embargo en veranos pocos lluviosos el embalse se estratifica de manera tal que sólo se rompe la termoclina cuando ocurren intensas lluvias.

El manejo del embalse provoca que las mayores concentraciones de nutrientes se extraigan por la toma destinada para el consumo humano.

La gestión del agua embalsada es una necesidad para evitar procesos de sedimentación en el 
embalse, así como el aumento de las concentraciones de fósforo y nitrógeno

\section{AGRADECIMIENTO}

Los autores agradecen el apoyo de Vicente Sánchez, técnico de la empresa Hidráulica Paso Bonito para la realización de este trabajo. Igualmente, los autores quieren expresar su agradecimiento al Profesor W. A. Wurtsbaugh por las sugerencias realizadas en el manuscrito y que han mejorado substancialmente el alcance de los resultados obtenidos.

\section{BIBLIOGRAFÍA}

AL BAKRI, D. \& M. CHOWDHURY. 2006. Internal nutrient flux in an inland water supply reservoir, New South Wales, Australia. Lakes \& Reservoirs: Research and Management, 11: 39-45.

APHA-AWWA-WPCF. 1998. Standard methods for the examination of water and wastewater. $20^{\text {th }} \mathrm{ed}$. Washington, DC. 22648 pp.

BETANCOURT, C., R. SUAREZ \& L. TOLEDO. 2009. Patrones de distribución de variables físicoquímicas en el embalse Paso Bonito, Cienfuegos, Cuba. Limnetica, 28: 23-34.

BEUTEL, M. W. 2006. Inhibition of ammonia release from anoxic profundal sediments in lakes using hypolimnetic oxygenation. Ecological Engineering, 28: 271-279.

BEUTEL, M. W., A. J. HORNE, W. D. TAYLOR, R. F. LOSEE, \& R. D. WHITNEY. 2008. Effects of oxygen and nitrate on nutrient release from profundal sediments of a large, oligo-mesotrophic reservoir, Lake Mathews, California. Lake and Reservoir Management. (In press).

CASTAGNINO, W. A. 1982. Investigación de modelos simplificados de eutroficación en lagos tropicales. Organización Panamericana de la Salud, Centro Panamericano de Ingeniería Sanitaria y Ciencias del Ambiente. Versión revisada. 27 pp.

CEPIS (Centro Panamericano de Ingeniería Sanitaria y Ciencias Ambientales). 2001. Metodología simplificada para la evaluación de eutrofización en lagos cálidos tropicales. Programa Regional. CEPIS/HPE/OPS. 63 pp.
CHOWDHURY, M. \& D. AL BAKRI. 2006. Diffusive nutrient flux at the sediment-water interface in Suma Park Reservoir, Australia. Hydrological Sciences Journal, 51(1): 144-156.

COMAS, A., A. MOREIRA \& S. URIZA. 2007. Cianoprocariotas (Cianobacterias, Cianoficeas) y microalgas como indicadoras de la calidad del agua en el embalse Paso Bonito Cienfuegos, Cuba. Proyecto Ramal Agencia Medio de Ambiente, CITMA, 39 pp.

DILLON, P. J. \& F. H. RIGLER. 1975. A simple method for predicting the capacity of a lake for development base on lake trophic status. Can. Board J. Fish. Res., 32: 1519-1531.

DOWNING, A. L. \& E. MCCAULEY. 1992. The nitrogen:phosphorus relationship in lake. Limnol. Oceanogr., 37: 936-945.

FOY, R. H. 1992. A phosphorus loading model for Northern Irish Lakes. Water Res., 26: 633-638.

JAMES, C., J. FISHER, \& B. MOSS. 2003. Nitrogen driven lakes: The Shropshire and Cheshire Meres? Arch. Hydrobiol., Arch. Hydrobiol., 158: 249-266.

KNOX, S., D. R. TURNER, A. G. DICXSON, M. I. LIDDICOAT, M. WHITFIELD, \& E. I. BUTLER. 1981. A statically analysis estuarine profile: A application to manganese and ammonium in the Tarner estuary. Estuarine Coastal and Shell Science, 13: 357-371.

MABERLY, S. C., L. KING, C. E. GIBSON, L. MAY, R. I. JONES, M. M. DENT, \& C. JORDAN (2003). Linking nutrient limitation and water chemistry in upland lakes to catchment characteristics. Hydrobiologia, 506: 83-91.

MORRIS, D. P. \& W. M. LEWIS. 1988. Phytoplankton nutrient limitation in Colorado mountain lakes. Freshwater. Biol., 20: 315-327.

NADIM, F., G. E. HOAG, F. L. ORDEN, G. S. WARNER, \& A. C. BAGTZOGLOU. 2007. Water quality characteristics of two reservoir lakes in eastern Connecticut, USA. Lakes \& Reservoirs: Research and Management, 12: 187-202.

NC (NORMA CUBANA). 1986. Fuentes de abastecimiento de agua. Higiene Comunal. Calidad y protección Sanitaria. Oficina Nacional de Normalización. República de Cuba. 23 pp.

PALAU, A. 2003. Medidas de gestión y adecuación ambiental de embalses frente a la eutrofia. Limnetica, 22(1-2): 1-13.

SALMASO, N., R. MOSELLO, L. GARIBALDI, F. DECET, M. C. BRIZZIO, \& P. CORDELLA. 2003. Vertical mixing as a determinant of trophic 
status in deep lakes: a case study from two lakes south of the Alps (Lake Garda and Lake Iseo). $J$. Limnol., 62 (Suppl. 1): 33-41.

SÁNCHEZ, R. 2000. Estudio del embalse Hanabanilla. Informe Científico Técnico. Delegación Provincial de Recursos Hidráulicos de Villa Clara, Cuba, $72 \mathrm{pp}$.

SEO, D. \& R. CANALE. 1999. Analysis of sediments characteristic and total phosphorus models for Shagawa lake. Journal of Environmental Engineering, 125(4): 346-350.

SIVADIER, F., J. M. THEBAULT, \& M. J. SALEN-
CON. 1994. Total phosphorus budget in Pareloup reservoir. Hydroecol. Appl., 6: 115-138.

STRASKRABA, M., I. DOSTÁLKOVÁ, J. HEJZLAR, \& V. VYHNÁLEK. 1995. The effect of reservoirs on phosphorus concentration. Int. Revue ges. Hydrobiol., 80: 403-413.

VOLLENWEIDER, R. A. 1969. Möglichkeiten und Grenzen elementarer Modelle der stoffbilanz Von Seen. Arch. Hydrobiol., 66: 1-36.

WETZEL, R. G. 2001. Limnology: Lake and river ecosystems. $3^{\text {rd }}$ Edition. Academic Press, San Diego. EEUU. 1006 pp. 